\title{
ARC LENGTH DIFFERENCE METHOD IN THE SELECTION OF PRIMARY SOURCES OF COMBINED EXTRAGALACTIC RADIO SOURCE CATALOGUES
}

\author{
J. L. LI ${ }^{1}$ and W. J. JIN ${ }^{2}$ \\ 1 Yunnan Observatory, Kunming 650011, P. R. China \\ ${ }^{2}$ Shanghai Observatory, Shanghai 200030, P. R. China
}

\begin{abstract}
The coordinates of common extragalactic radio sources will be different if the orientations of individual frames are different. If coordinates in each of the frames are in good consistence, i.e. small or non local relative deformations exist, common arcs in frames will be equal to each other (within precision) whether the orientations of the frames coincide or not. In addition, if relative deformations exist between frames, these deformations will be reflected partly on the difference of the lengths of common arcs. It is inferred that we can select candidates for primary objects by comparing the lengths of common arcs in individual frames. We call this as the Arc Length Difference (ALD) selection method.
\end{abstract}

Considering that it is unrealistic to distinguish between positions with or without deformations beforehand (Arias et al., 1988), and that an arc length difference gives us nothing definite about the consistency of a specified source in frames because of the variable values and the statistical zero mean of the differences ( $\mathrm{Li}$ \&in, 1994), we take the Mean value of all the Absolute Arc Length Differences (MAALD) related to each specified source as the criterion for the recognition of deformation. The large the deformation for a particular source is, the larger (statistically) the absolute value of the arc length difference or the MAALD related to this source will be. Taking all the MAALDs of all the common objects of two frames as a random series, and deleting large MAALDs in this series by means of statistics, we can select candidates for primary objects which have better internal and external consistencies in frames.

Here we make emphasis on the following two aspects of the ALD method. First, if we take all the absolute values of the arc length differences as a random series and perform statistical deletion on this series, there will come out the situation that among all the absolute values related to a specified source some will be deleted and others will be kept because of the variable value nature of the arc length difference. Therefore the characteristics of consistency of the source will be concealed and we will be faced to the ambiguous case to decide whether the source should be deleted or not. The effect of the variable value nature of the arc length difference can be avoided and the characteristics of consistency of a source is highlighted by using the mean of all the absolute values related to this source. Second, since the value of MAALD is the mean of all the absolute arc length differences related to a source, a specified arc length difference will enter into two MAALDs, so that all the MAALDs are then interweaved with one another. After one of the MAALDs is deleted, the rest will be changed accordingly. So we emphasize a progressive process to avoid a large number of sources being deleted in a single step.

Our tests show that the ALD selection method is stable and reliable. It can emphasize the characteristics of each source on the premise of the consistency of all the sources and the selection results of this method can reflect appropriately the total number of sources, the position precision, the sky coverage and so on of individual frames.

\section{Reference}

Arias, E.F., Feissel, M, Lestrade, J.-F., 1988, In: BIH Annual Report for 1987, D-113

Li, J.L. \& Jin, W.J., 1994, Annals of Shanghai Observatory, Academia Sinica, No.15, 98 